\title{
Magnetically driven dielectric and structural behaviour in $\mathrm{Bi}_{0.5} \mathrm{La}_{0.5} \mathrm{FeO}_{3}$
}

\author{
Christopher M. Kavanagh, ${ }^{1}$ Richard J. Goff, ${ }^{1}$ Aziz Daoud-Aladine, ${ }^{2}$ Philip Lightfoot, ${ }^{1}$ Finlay D. Morri- \\ son $^{1 *}$
}

1. School of Chemistry and EaStCHEM, University of St Andrews, St Andrews, KY16 9ST, United Kingdom

2. ISIS Facility, Rutherford Appleton Laboratory-STFC, Chilton, Didcot, Oxfordshire OX11 0QX, United Kingdom

KEYWORDS BiFeO ${ }_{3}$, Perovskite, Octahedral tilt, Magnetostriction, A-site displacement, Multiferroic, Dielectric

\begin{abstract}
A detailed structural analysis of the antiferromagnetic ( $\mathrm{G}_{2}$-type) lanthanum doped bismuth ferrite - $\mathrm{Bi}_{0.5} \mathrm{La}_{0.5} \mathrm{FeO}_{3}$ $\left(P n^{\prime} m a^{\prime}\right)$ - using variable temperature powder neutron diffraction is reported. The analysis highlights a structural link between changes in the relative dielectric permittivity and changes in the $\mathrm{FeO}_{6}$ octahedral tilt magnitudes, accompanied by a structural distortion of the octahedra with corresponding A-site displacement along the c-axis; this behaviour is unusual due to an increasing in-phase tilt mode with increasing temperature. The anomalous orthorhombic distortion is driven by magnetostriction at the onset of antiferromagnetic ordering resulting in an Invar effect along the magnetic c-axis and anisotropic displacement of the Asite $\mathrm{Bi}^{3+}$ and $\mathrm{La}^{3+}$ along the a-axis.
\end{abstract}

\section{INTRODUCTION}

$\mathrm{BiFeO}_{3}$ is a widely studied multiferroic due to its ferroelectric (high $\mathrm{T}_{\mathrm{C}} \sim 1083-1103 \mathrm{~K}$ ) and (anti)ferromagnetic properties $\left(T_{\mathrm{N}} \sim 643 \mathrm{~K}\right) .^{1}$ However, applications and the study of bulk $\mathrm{BiFeO}_{3}$ are hindered by electrical conductivity due to nonstoichiometry and thermal metastability. ${ }^{2}$ The electrical properties and thermal stability of the perovskite phase can be improved by the substitution of the volatile $\mathrm{Bi}^{3+}$ with rare earths e.g. $\mathrm{La}^{3+}\left(\mathrm{Bi}_{1-x} \mathrm{La}_{x} \mathrm{FeO}_{3}\right)$.

The substitution of $\mathrm{Bi}^{3+}$ with $\mathrm{La}^{3+}$ results in a diverse range of phases at lower doping levels (i.e., $x<0.5$ ), which has been reported in a number of studies including those of Rusakov et $a l^{3}$ and Troyanchuk et $a .^{4}$ The experimental observations have been supported by first principles studies showing that several of the $\mathrm{Bi}_{1-x} \mathrm{La}_{x} \mathrm{FeO}_{3}$ phases are quasidegenerate in energy over a wide compositional range. ${ }^{5}$ This series of phase mixtures complicates the study of the materials at lower doping levels $(x<0.5)$ and has driven the current study to review compositions at higher $\mathrm{La}^{3+}$ content.

Rusakov et al. observed the following phase sequence (under ambient conditions) as a function of composition for samples prepared by solid-state methods: $R 3 c(0<\mathrm{x}<0.1) \rightarrow$ 'Pnam $^{\prime}(\mathrm{x}$
$=0.18) \rightarrow$ incommensurate 'Imma' $(0.19<x<0.30) \rightarrow$ Pnma $(x>0.5) .{ }^{3}$ More specifically, the 'Pnam' phase has a $\mathrm{PbZrO}_{3}$ like antipolar superlattice, with a $\sqrt{2} a_{p} \times 2 \sqrt{2} a_{p} \times 4 a_{p}$ unit cell, relative to the aristotype cubic perovskite $\left(a_{p}\right)$, the 'Im$m a^{\prime}$ phase approximates to a $\sqrt{2} a_{p} \times 2 a_{p} \times \sqrt{2} a_{p}$ unit cell, and the Pnma structure is the common $\mathrm{GdFeO}_{3}$-type structure, with a $\sqrt{ } 2 a_{p} \times 2 a_{p} \times \sqrt{ } 2 a_{p}$ unit cell.

Two-phase regions exist over a wide range of compositions between the single-phase regions defined above, and the boundaries of the phase fields vary depending on the study and synthesis conditions used. For example, the anitpolar $\mathrm{PbZrO}_{3}$-like phase has been studied $(x=0.185)$ as a phase composition that initially adopts the $R 3 C$ space group and transforms isothermally to the antipolar structure. ${ }^{6}$ Electron and $\mathrm{x}$-ray diffraction studies of $\mathrm{x}=0.25$ showed that the samples comprised of coarse and fine grains of the nominal compositions $x=0.1$ and $x=0.45$ respectively. ${ }^{7}$ Increasing the synthesis temperature, it was found that the composition of the grains changed to a situation where finer grains were rich in bismuth and iron and no longer contained $\mathrm{La}^{3+}$ ions and the coarse phase remained. ${ }^{7}$

In addition to variations due to synthesis conditions, structural characterisation of some of the phases can prove challeng- 
ing. For instance the 'Pnam' phase, which has been successfully applied to other lanthanide dopants, has been suggested to contain a more complex octahedral tilt system, leading to a quadrupled axis, perhaps analogous to that seen in $\mathrm{NaNbO}_{3} .^{8-}$ 10 This additional distortion, however, is not sufficiently strong and/or ordered to give clear superlattice reflections in conventional $\mathrm{x}$-ray or neutron diffraction experiments, and the $\mathrm{PbZrO}_{3}$-like structure, ${ }^{11}$ space group Pbam, has been used as a model for neutron powder diffraction data. ${ }^{6}$

To understand why this complex sequence of phases is observed, changes in the crystal structure as a function of composition must be fully understood. These structural changes affect the properties of the material and occur, in large part, due to the electronic difference between $\mathrm{Bi}^{3+}$ and $\mathrm{La}^{3+}$ i.e., the presence of the $\mathrm{Bi}^{3+}$ lone pair. The effect of substituting $\mathrm{Bi}^{3+}$ by $\mathrm{La}^{3+}$ on the A-site has been considered in a number of different studies. Density functional theory (DFT) calculations, corroborated by experimental evidence, suggest that within the $R 3 c$ phase the degree of the ferroelectric off-centering along the c-axis of the unit cell (hexagonal setting) is reduced with increasing lanthanum content. ${ }^{12}$ This influences the ferroelectric properties, significantly reducing the ferroelectric polarisation with doping concentration. However, it does allow samples to sustain higher electric fields by reducing leakage currents. ${ }^{12}$

The local, short-range magnetic order of $\mathrm{BiFeO}_{3}$ is a G-type antiferromagnet with a weak canted moment. ${ }^{1}$ However, the overall long-range magnetic order is more complicated and is based on an incommensurate spin cycloid of the antiferromagnetic sub-lattice. ${ }^{1}$ The cycloid structure propagates along the [110] direction and has a repeat distance of about 62-64 $\mathrm{nm} .{ }^{13,14}$ This repeat distance can be influenced by changes on the A-site. Le Bras et al. show that in polycrystalline samples, lanthanum substitution within the $R 3 c$ phase $(x>0.15)$ results in a destabilising effect on the spiral magnetic structure and an increased predisposition to change to a collinear magnetic structure. ${ }^{15}$

Another example of the effect of A-site substitution is the observation of the incommensurate 'Imma' phase $(x=0.25)$ which has been suggested based on a synchrotron powder $X$ ray diffraction study. ${ }^{3}$ The incommensurate modulation is suggested to be due to competing dipole-dipole interactions caused by antipolar displacements of $\mathrm{Bi}^{3+}$ and octahedral tilting. ${ }^{3}$ Anisotropic refinements of the Imma phase suggest a displacement along the b-axis. ${ }^{3}$

The magnetic transition appears to be fairly temperature independent across the compositional range $0.18 \leq x \leq 0.3$. Changes associated with a transformation in the magnetic behaviour have been detected using differential scanning calorimetry (DSC). Rusakov et al. reported a weak anomaly near $670-675 \mathrm{~K}(0.18 \leq \mathrm{x} \leq 0.35)$ and suggested it was associated with the antiferromagnetic to paramagnetic phase transition ${ }^{3}$ that has been reported elsewhere by Roginskaya and Polomska. ${ }^{16,17} \mathrm{LaFeO}_{3}$ is a canted G-type antiferromagnetic phase with a slightly off axis component ${ }^{3,18}$ and with a $T_{N}$ of $743 \mathrm{~K}^{19,20}$
A number of authors report that above a critical concentration ( $x \gtrsim 0.5$ ) the Pnma structure is adopted. ${ }^{3,4}$ This is the $\mathrm{GdFeO}_{3}$-type structure adopted by the parent $\mathrm{LaFeO}_{3}$ phase and indeed all rare earth orthoferrites. ${ }^{21}$ It is also the structure adopted by $\mathrm{BiFeO}_{3}$ in the high temperature paraelectric phase. ${ }^{22,23}$ The Pnma structure, however, is commonly refined in the alternate crystallographic setting Pbnm. ${ }^{18,19,24}$

The electrical properties of the $\mathrm{Bi}_{1-\mathrm{x}} \mathrm{La}_{\mathrm{x}} \mathrm{FeO}_{3}$ system remain to be studied in detail in relation to the structural behaviour. Typically, authors have found that the conduction process of the bulk is dominated by the presence of double ionised oxygen vacancies. ${ }^{25,26}$ High conductivities, however, mean that obtaining evidence for structural transitions from electrical measurements is impossible.

In this study, new findings are presented based on temperature dependent powder neutron diffraction (PND) and electrical impedance spectroscopy data on carefully prepared electrically insulating samples. The current work focusses on the composition $\mathrm{Ba}_{1-\mathrm{x}} \mathrm{La}_{x} \mathrm{FeO}_{3}, \mathrm{x}=0.5$ (BLFO50) and reveals an unusual structural response with temperature involving an increasing in-phase octahedral tilt with increasing temperature, linked to magnetostriction. In addition, it is possible to demonstrate a correlation between structural phenomena and the observed changes in the dielectric behaviour. These structural changes relate to correlated displacements of the A-site cations and octahedral distortion and tilting.

\section{EXPERIMENTAL METHODS}

The synthesis was performed via conventional solid-state methods. Stoichiometric amounts of $\mathrm{Bi}_{2} \mathrm{O}_{3}$ and $\mathrm{Fe}_{2} \mathrm{O}_{3}$ were mixed with $\mathrm{La}_{2} \mathrm{O}_{3}$ (dried at $1000{ }^{\circ} \mathrm{C}$ prior to weighing) in an agate mortar and pestle. The mixed powders were ball milled in ethanol using a Fritsch Pulverisette 7 planetary micro ball mill (600 rpm, $1 \mathrm{hr}$ ). The dry powders were calcined in a twostep reaction under an enriched nitrogen atmosphere. In the initial step, pelletized samples were heated to $800{ }^{\circ} \mathrm{C}$ in an alumina boat lined with platinum foil, where they remained at temperature for $5 \mathrm{hrs}$. The reground pellets were milled under the same milling conditions prior to the second heating. The loose powder was pelletized in a uniaxial press using a $13 \mathrm{~mm}$ stainless steel die to which a pressure of $c a$. $70 \mathrm{MPa}$ was applied to the pellet and sintered at $1000{ }^{\circ} \mathrm{C}$ for a further 5 hrs.

These pellets were used for ac impedance measurements; silver paste electrodes were applied and cured at $120^{\circ} \mathrm{C}$. Temperature control at elevated temperatures was controlled using a Carbolite furnace. Sub-ambient temperature control was based on a Sumitomo HC-2 cryogenics system. Isothermal measurements were taken in a conductivity jig fabricated with platinum wire contacts and a K-type thermocouple in close proximity to the pellet. High temperature isothermal measurements were taken using a Hewlett Packard 4192A LF impedance analyser. The temperature sweep data were collected using Agilent 4294A (sub ambient) and Wayne Kerr 6500B (high temperature) impedance analysers. All data were corrected for sample geometry. 
PND experiments were performed on the HRPD diffractometer at the ISIS neutron spallation source using two different sample environments. The experimental temperature range was from $50 \mathrm{~K}$ to $900 \mathrm{~K}$ : measurements from $50 \mathrm{~K}$ to $550 \mathrm{~K}$ were carried out in a cryofurnace and those from $450 \mathrm{~K}$ up to $900 \mathrm{~K}$ were performed in a furnace, resulting in some temperature overlap. Patterns were collected at $450 \mathrm{~K}, 500 \mathrm{~K}, 550$ $\mathrm{K}$ in the furnace and $550 \mathrm{~K}$ in the cryofurnace allowing for a comparison between the different sample environments. Rietveld refinements were carried out using the general structural analysis system (GSAS) and its associated graphical user interface program (EXPGUI). ${ }^{27,28}$ Only data from the 'backscattering' detector bank $\left(2 \theta \sim 168^{\circ}\right)$ on the HRPD instrument was used for refinements $(0.7<d<2.6 \AA$ ) , although $30^{\circ}$ bank data was examined for evidence of persistence of magnetic ordering.

\section{RESULTS}

\section{Preliminary structural analysis}

The PND data show that BLFO50 occurs predominantly as the Pnma, $\mathrm{GdFeO}_{3}$-type phase (94 wt\% at room temperature). The final Rietveld refinement at $300 \mathrm{~K}$ is shown in Figure 1. The major phase has been refined with a G-type magnetic structure with the moment constrained along the c-axis, described with the Shubnikov symmetry $P n^{\prime} m a^{\prime}$. The possibility of a minor off axis magnetic component which is typically found in rare earth orthoferrites ${ }^{29}$, however, should not be excluded (attempts to refine any off-axis magnetic component were unsuccessful; direct measurement of a net moment due to spin canting using conventional ac susceptibility measurements is hampered by the trace presence of ferromagnetic hematite). The minor secondary phase ( $<5 \mathrm{wt} \%)$ is an I-centred phase, which transforms to Pnma as a function of temperature (see Supporting Information, Figure $\mathrm{S} 1$ ). This phase has been refined as tetragonal, $14 / \mathrm{mcm}\left(a=b \sim \sqrt{2} \mathrm{a}_{\mathrm{p}}, c\right.$ $\sim 2 a_{p}$ ) although it is probably the incommensurate orthorhombic ('Imma') phase reported by Rusakov et al. ${ }^{3}$ Exact and definitive identification of the space group is difficult as the nuclear reflections from this secondary phase are predominantly observed only as broad shoulders at the base of the $P n^{\prime} m a^{\prime}$ reflections; any magnetic structure peaks associated with this phase are unlikely to be detectable. This secondary phase is no longer present above circa $650 \mathrm{~K}$, transforming to Pnma. The trace amount of $\mathrm{Fe}_{2} \mathrm{O}_{3}$ (ca. 1 wt\%) remains constant in all refinements. The unit cell parameters and atom positions for the main $P n^{\prime} m a^{\prime}$ phase at $300 \mathrm{~K}$ are shown in Table 1. The Rietveld refinement (Figure 1 ) at $300 \mathrm{~K}$ confirms the need for the magnetic model (see Supporting Information, Figure S2).

\begin{tabular}{lllll} 
Site & $\mathbf{X}$ & $\mathbf{Y}$ & $\mathbf{Z}$ & $\mathbf{U}_{\text {iso }}\left(\AA^{2}\right)$ \\
\hline $\mathrm{Bi} / \mathrm{La}$ & $0.0233(3)$ & 0.25 & $-0.0072(7)$ & $0.0199(3)$ \\
$\mathrm{Fe}$ & 0 & 0 & 0.5 & $0.00209(16)$ \\
$\mathrm{O} 1$ & $0.4872(5)$ & 0.25 & $0.0741(6)$ & $0.0118(3)$ \\
$\mathrm{O} 2$ & $0.2835(3)$ & $0.0409(3)$ & $-0.2834(3)$ & $0.0118(3)$ \\
\hline
\end{tabular}

Table 1. Atomic coordinates of the $P n^{\prime} m a^{\prime}$ phase of $\mathrm{Bi}_{0.5} \mathrm{La}_{0.5} \mathrm{FeO}_{3}$ refined from PND data at $300 \mathrm{~K} ; \mathrm{a}=5.5749$ (8) $\AA, \mathrm{b}=7.8653(1) \AA$, $c=5.5551(1) \AA$.

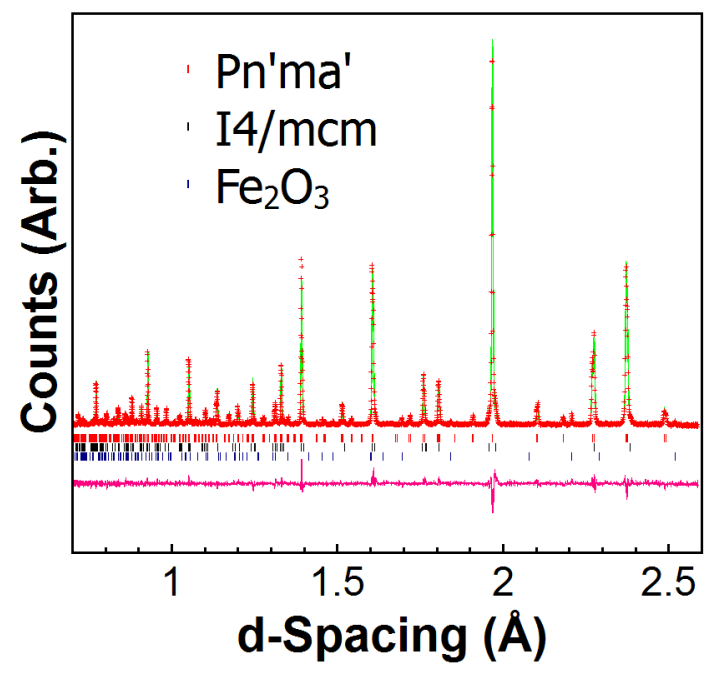

Figure 1. Rietveld refinement profile of PND data at $300 \mathrm{~K} ; \chi^{2}=$ 5.157, $w R p=0.06, R p=0.0544$. Tick marks represent Braggs reflections for $P n^{\prime} \mathrm{ma}^{\prime}$ (top) and $14 / \mathrm{mcm}$ (middle) $\mathrm{Bi}_{0.5} \mathrm{La}_{0.5} \mathrm{FeO}_{3}$ phases and $\mathrm{Fe}_{2} \mathrm{O}_{3}$ (bottom).

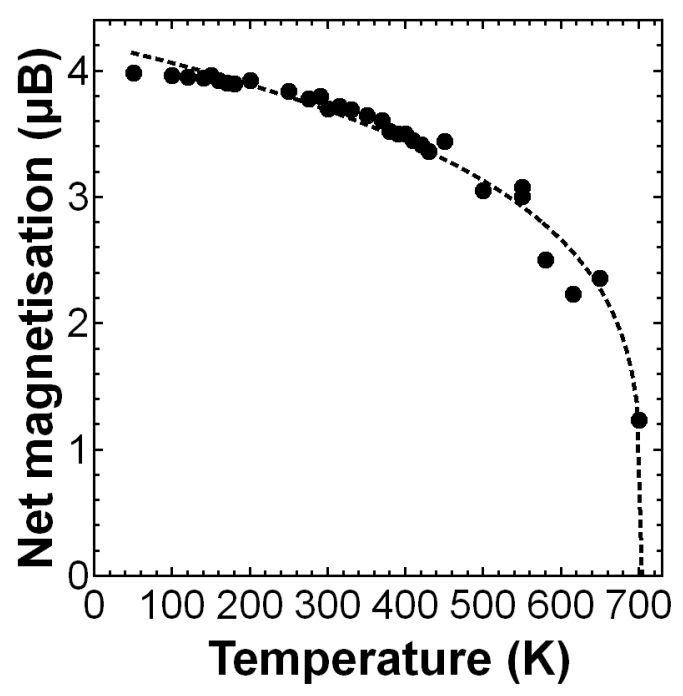

Figure 2. Net magnetisation (along c) as a function of temperature $(\mathrm{K})$ as determined from Rietveld refinement of PND data.

The net magnetic moment, as determined from refinement of the PND data, decays with temperature (Figure 2). The data was fitted using a power law, $F(T) \propto\left(T_{N^{-}} T\right)^{\alpha}$, assuming a mean field approximation and gives a Neel temperature, $\mathrm{T}_{\mathrm{N}}=704 \pm$ $3 \mathrm{~K}$ and exponent $\alpha=0.24 \pm 0.04$. This is supported by the persistence of magnetic reflections up to $700 \mathrm{~K}$ (Supporting Information, figure S3) and is consistent with previously re- 
ported values for this composition and the parent $\mathrm{LaFeO}_{3}$ phase $\left(T_{N} \sim 743 \mathrm{~K}\right) \cdot{ }^{16,17,19,20}$

The unit cell parameters (Figure 3 ) clearly show an increasing orthorhombic distortion with increasing temperature up to ca. $700 \mathrm{~K}$ due to the invariant $\mathrm{c}$-axis relative to the expansion of $a$ and $b$. This orthorhombic strain can be represented by the distortion parameter, $D_{o}$, defined as:

$$
D_{o}=\frac{|a-c|}{2(a+c)}
$$

Above ca. $700 \mathrm{~K}$, the $\mathrm{c}$ axis increases and $D_{0}$ decreases (Figure 4.) This unusual behaviour of increasing orthorhombic strain with increasing temperature will be discussed further in light of the magnetic behaviour and the contributions of the various structural distortion modes allowed at both the A-site and involving the $\mathrm{BO}_{6}$ octahedra which are presented below.

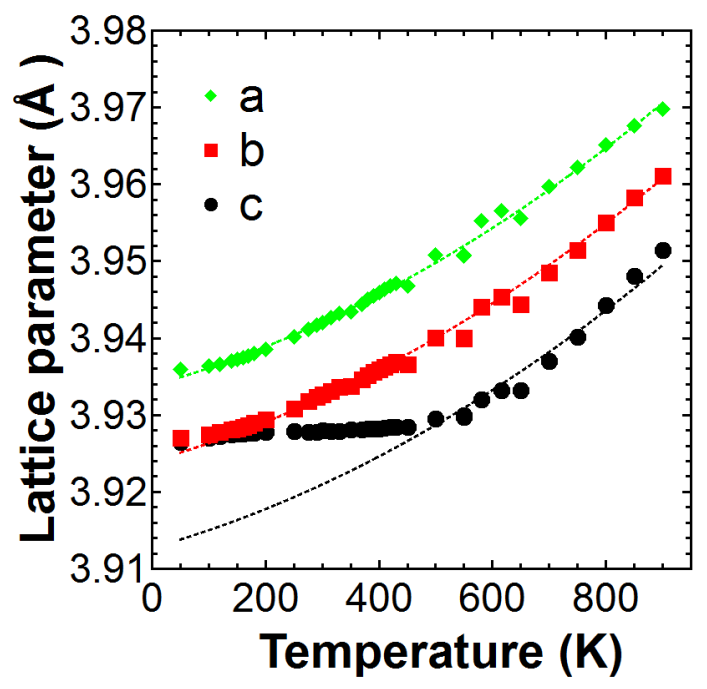

Figure 3. Reduced (psuedo cubic) cell lattice parameters as a function of temperature $\left(a=\sqrt{ } 2 a_{p}, b=2 a_{p}, c=\sqrt{ } 2 a_{p}\right.$. where $a_{p}$ represents lattice parameter of the cubic aristotype); dashed lines represent fit to a second order polynomial.

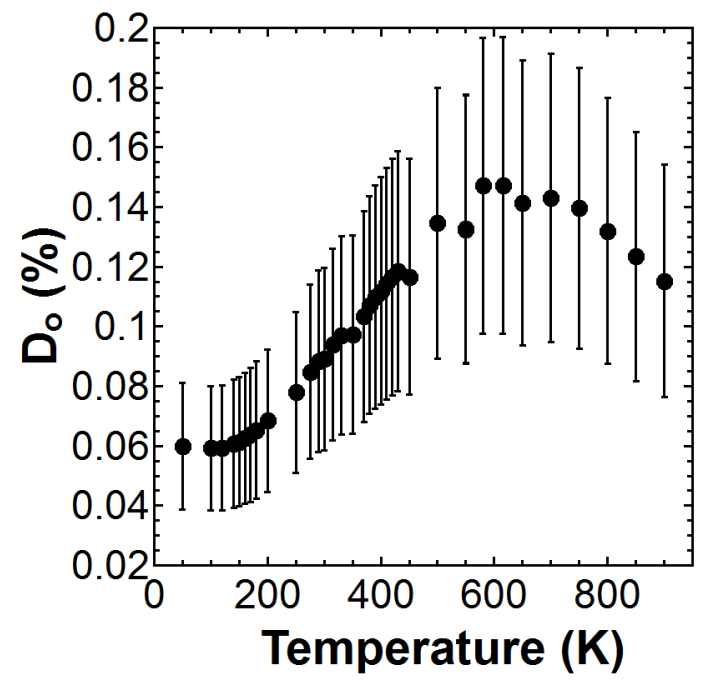

Figure 4. Orthorhombic strain, $D_{o}$, as a function of temperature.

\section{Electrical Properties}

The electrical properties of a ceramic are closely related to the microstructure and homogeneity. Typically the electrical microstructure of coarse polycrystalline ceramics is described by the brickwork model ${ }^{30,31}$ which essentially places each electroactive region (e.g. bulk, grain boundary, sampleelectrode interface) in a series combination. Each electroactive region is described by its resistive (R) and capacitive (C) behaviour which represents the various charge transfer and polarisation processes, respectively. The resulting equivalent circuit consists of a number of parallel RC elements placed in series, where each RC element describes a particular electroactive region. These regions have different magnitudes of associated $\mathrm{R}$ and $\mathrm{C}$ and $a c$ impedance spectroscopy separates the different electrical responses in the frequency domain according to their differing time constants, $\tau$ (where $\tau=R C$ ). Each RC element forms a semi-circular arc in the complex impedance $\left(\mathrm{Z}^{*}\right)$ and electric modulus $\left(\mathrm{M}^{*}\right)$ plane plot with the diameter of the arc proportional to the resistance and inverse capacitance, respectively. The individual RC elements also give rise to Debye-like peaks in the spectroscopic plots of the imaginary component ( $Z^{\prime \prime}$ or $M^{\prime \prime} v s$. frequency); the frequency at which the peak maximum, $f_{\max }$, occurs is determined by the time constant, equation 2.

$$
\tau=R C=\frac{1}{2 \pi f_{\max }}(2)
$$

While resistance values can vary dramatically and depend on a number of factors, there are typical magnitudes of capacitance associated with each type of electroactive region (as determined by relative permittivity and geometry). ${ }^{32}$ From the magnitude of associated capacitance, it is therefore possible to assign the various electrical responses to particular electroactive regions (e.g. bulk, grain boundary etc.).

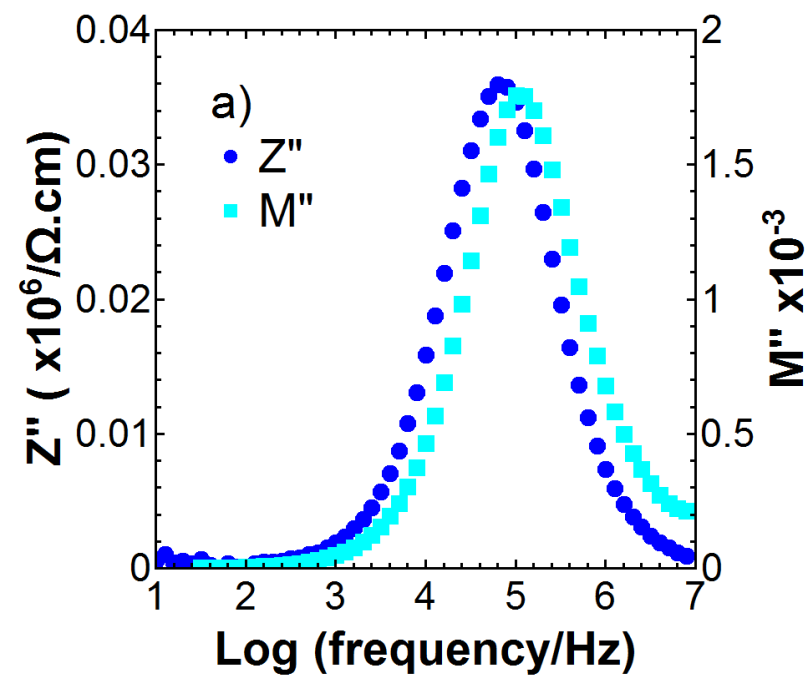



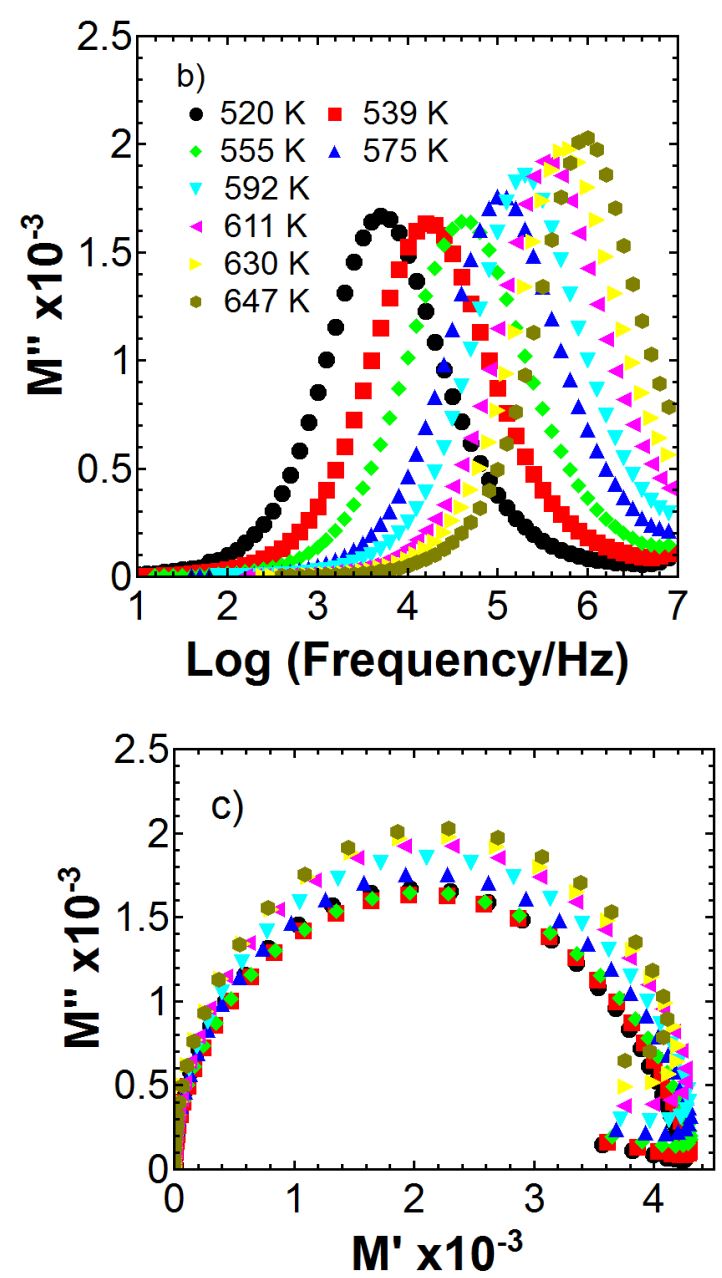

Figure 5. Imaginary electric modulus, $\mathrm{M}^{\prime \prime}$ and impedance, $\mathrm{Z}^{\prime \prime}$, spectroscopic plots at $575 \mathrm{~K}(\mathrm{a})$; $\mathrm{M}^{\prime \prime}$ spectra (b) and complex electric modulus, $M^{*}$ plane plots (c) as a function of temperature. (Symbols in part $c$ are as in b).

Typical magnitudes of capacitance for a grain boundary response are of the order $10^{-10}-10^{-8} \mathrm{Fcm}^{-1}$ compared to typical bulk values of $10^{-12}-10^{-11} \mathrm{Fcm}^{-1}$. $^{31}$

Ac impedance spectroscopy of insulating BLFO50 samples reveals only a single electroactive region as shown by the presence of a single Debye peak in both the imaginary modulus and impedance spectra (Figure 5a) and at the same (single) relaxation time (i.e., the peak maxima occur at the same frequency). Assuming Debye behaviour the modulus peak maximum, $\mathrm{M}^{\prime \prime}{ }_{\max }$, is related to the capacitance and dielectric permittivity by:

$$
M_{\text {max }}^{\prime \prime}=\frac{\varepsilon_{0}}{2 C}=\frac{1}{2 \varepsilon_{r}}
$$

From the magnitude of the $\mathrm{M}^{\prime \prime}$ peak this response has an associated $\mathrm{C}$ of $\mathrm{ca} .21 \mathrm{pFcm}^{-1}$ at $520 \mathrm{~K}^{2}$ and is therefore associated with the bulk response. ${ }^{31}$ With increasing temperature the $\mathrm{M}^{\prime \prime}$ peak increases in magnitude slightly indicating that the bulk capacitance is falling (Figure 5b); the peak is also displaced to higher frequency due to decreasing resistance, equation 2.
The permittivity of the bulk response can be determined more accurately from the diameter of the semi-circular arc in the complex electric modulus, $\mathrm{M}^{*}$, (Figure $5 \mathrm{c}$ ) which scales directly as $1 / \varepsilon_{\mathrm{r}}$. Permittivity data extracted in this manner is shown in Figure 6 along with data obtained from fixed frequency measurements (discussed below).

Fixed frequency capacitance $\left(C_{p}\right)$ and loss (tan $\left.\delta\right)$ measurements were performed on insulating samples of BLFO50. Based on the brick layer model and assuming an equivalent of two parallel RC elements in series (representing bulk and grain boundary regions) the parallel capacitance as a function of frequency results in two frequency independent plateaus. ${ }^{32}$ The parallel capacitance $\left(C_{P}\right)$ measured in the high frequency plateau contains contributions from both the bulk $\left(C_{b}\right)$ and grain boundary $\left(\mathrm{C}_{\mathrm{gb}}\right)$ capacitances according to:

$$
C_{P}=\left(C_{g b}^{-1}+C_{b}^{-1}\right)^{-1}
$$

The magnitudes of the capacitive responses expected in a coarse grained ceramic with thin, well-defined grain boundaries are such that $C_{g b}>>C_{b}$ and therefore the overall contribution of the grain boundary to the total capacitance is small. The total high frequency capacitance is therefore a close approximation of the bulk response. Provided high temperature data can be used to validate the assumption that $C_{g b}>>C_{b}$ then fixed frequency measurements at lower temperatures can be used to probe the bulk response. The single semi-circular bulk arc in $\mathrm{M}^{*}$, Figure $5 \mathrm{c}$, and the analysis described above indicates that $C_{g b} \gg C_{b}$ is indeed the case for our samples and so bulk permittivities were calculated from $\mathrm{C}_{\mathrm{p}}$ at $10 \mathrm{kHz}, 100 \mathrm{kHz}$ and $1 \mathrm{MHz}$ using the relationship:

$$
C_{P}=\varepsilon_{r} \varepsilon_{0} \frac{A}{d}
$$

where $A$ is the electrode area, $d$ is the separation between the electrodes (sample thickness), $\varepsilon_{0}$ is the permittivity of free space, and $\varepsilon_{r}$ the bulk relative permittivity. Bulk permittivity and loss data as a function of temperature are shown in Figure 6.

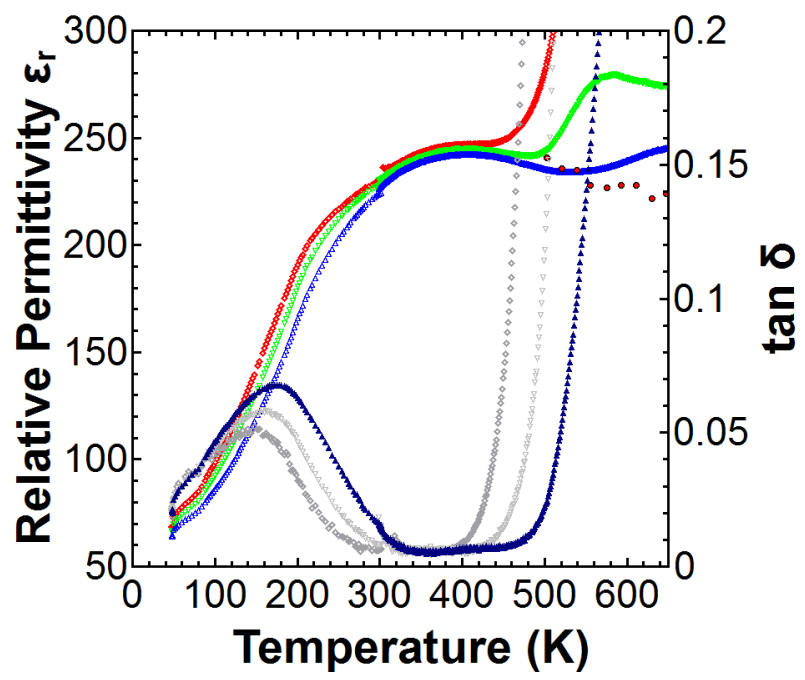


Figure 6. Relative dielectric permittivity $\left(\varepsilon_{r}\right)$ and loss ( $\left.\tan \delta\right)$ as a function of temperature at $10 \mathrm{kHz}, 100 \mathrm{kHz}$, and $1 \mathrm{MHz}$ (left to right at high temperature). Bulk values obtained from electric modulus data are included circles black outline.

The permittivity increases from ca. 60 at low temperature increasing to ca. 240 at $300 \mathrm{~K}$, the loss data exhibit a peak around $100 \mathrm{~K}$ (Figure 6). The data show only a weak frequency dispersion over the majority of the temperature range; the dramatic upturn in the data at the highest temperatures (particularly evident in the dielectric loss) is due to the increasing contribution of the grain boundary/electrode response and is not intrinsic. Bulk permittivities determined from $\mathrm{M}^{*}$ plots, Figure 5c confirm this and indeed show that in fact the bulk permittivity decreases slightly above $500 \mathrm{~K}$. This dielectric behaviour must be intrinsically linked to some structural change, which is not immediately obvious from the data presented in Figure 3. A more in-depth structural analysis is required.

Bulk conductivities, $\sigma_{b}\left(=R_{b}{ }^{-1}\right)$ were estimated using two methods: bulk resistances $\left(R_{b}\right)$ were obtained from $M^{\prime \prime}$ maxima and also from complex impedance plane plots using the relationships in equation 2 and 3 (see also Supporting Information, Figure S4) The conductivities determined by each method are in good agreement, with both showing the expected temperature dependence (Figure 7) described by the Arrhenius expression:

$$
\sigma_{b}=\sigma_{0} \exp ^{-\frac{E a}{k T}}
$$

The bulk activation energy $\left(E_{a}\right)$ is $\sim 1.2 \mathrm{eV}$ which is consisted with other experimental studies ${ }^{26,33}$ and is characteristic of electronic conduction dominated by the presence of oxygen vacancies. $^{34}$

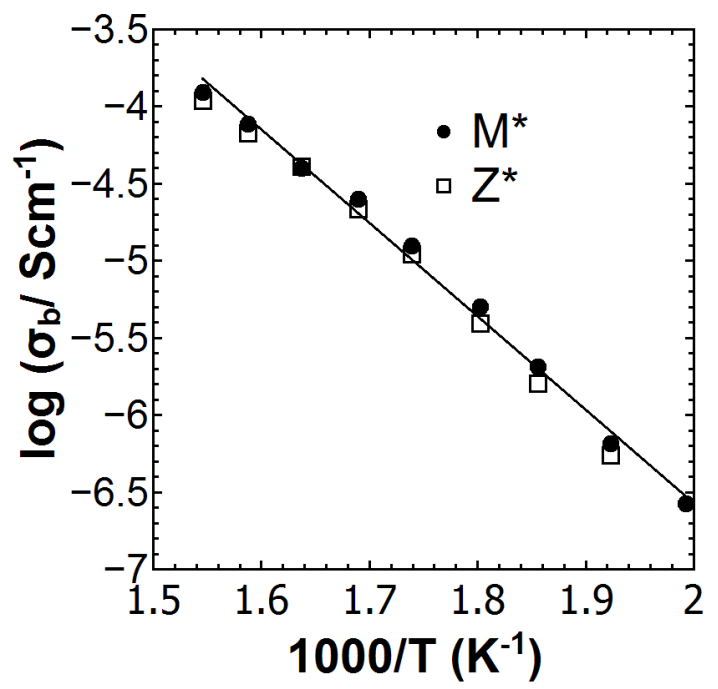

Figure 7. Arrhenius plot of bulk conductivities determined form both $Z^{\prime *}$ and $M^{*}$ data; activation energy, $E a=1.20 \mathrm{eV}\left(R^{2}=0.992\right)$

\section{Correlation of structure and dielectric response}

In order to correlate the change in dielectric response (Figure 6) with crystallographic changes the crystal structures as de- rived from Rietveld refinements were analysed both in terms of a conventional 'bond length/bond angle' description and also using the online structural analysis tool ISODISTORT. ${ }^{27,35}$ The latter allows comparison of a distorted structure with its parent aristotype (an ideal, cubic perovskite in this case) in terms of specific symmetry-allowed modes which are derived from irreducible representations of the parent symmetry. The advantage of this method is that it allows both the nature and degree of each particular distortion mode to be determined in a completely decorrelated manner - for example, 'octahedral tilt' modes and 'cation displacement' modes can be seen independently. The seven variable atomic coordinates (Table 1) can therefore equivalently be represented by seven distinct symmetry-adapted modes.

The initial Rietveld analysis showed that the $\mathrm{FeO}_{6}$ octahedra become more regular in terms of Fe-O bond lengths up to $\mathrm{ca}$. $300 \mathrm{~K}$ (Figure 8); at the same time, however, the internal octahedral angles (O-Fe-O angles, Supporting Information, Figure S5) show deviation from an ideal octahedron.

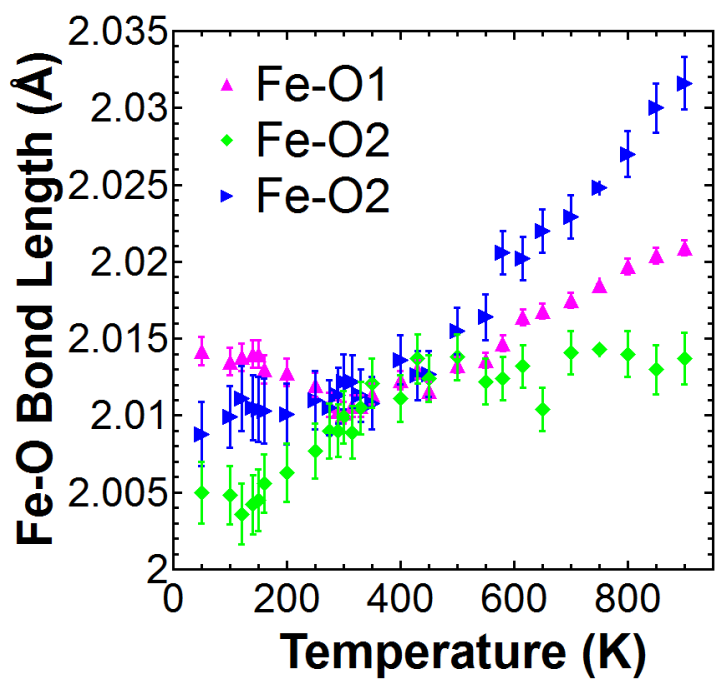

Figure 8. Fe-O bond lengths as a function of temperature as determined from Rietveld refinements of PND data.

This distortion can be represented clearly using the ISODISTORT software, where it is described by the $\mathrm{R}_{5}{ }^{+}$modes relating to the $A$-site and oxygen positions. The $R_{5}{ }^{+}$[A site] mode represents a displacement of the A-site cation along the c-axis, Figure 9. In terms of the degree of distortion these modes should be viewed as absolute magnitudes and therefore the $\mathrm{R}_{5}{ }^{+}$[A site] at $300 \mathrm{~K}$ represents the most distorted state relative to the ideal model. This corresponds to the broad maximum in the relative permittivity (Figure 6) 


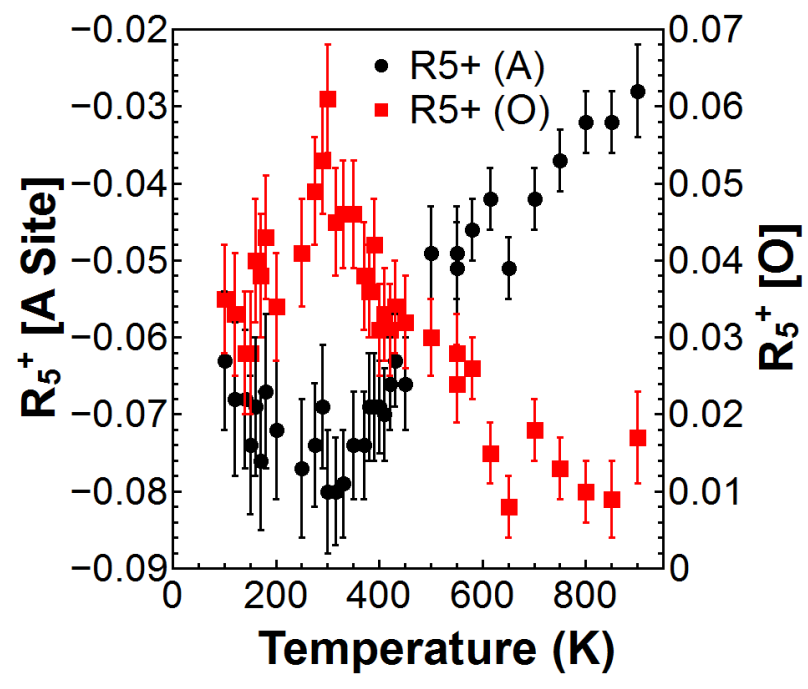

Figure 9. ISODISTORT displacement $\left(\mathrm{R}_{5}{ }^{+}\right)$modes as a function of temperature showing the A-Site displacement along $c$ axis $\left(R_{5}{ }^{+}[A\right.$ site]) and displacements of the axial oxygens (O1) along $c$ and the equatorial oxygen (O2) along $b\left(\mathrm{R}_{5}{ }^{+}[\mathrm{O}]\right)$.

This A-site displacement also correlates with the $\mathrm{R}_{5}{ }^{+}$[O] mode, which is a 'scissoring' distortion of the $\mathrm{FeO}_{6}$ octahedra (where the axial oxygens (O1) are allowed to move along $c$ while the equatorial oxygens (O2) displace along b). This means that while the octahedra are becoming more regular in terms of bond lengths, they are increasingly distorted in terms of O-Fe-O angles. This distortion, together with the displacement on the A-site leads to an increase in the relative permittivity. This higher than expected permittivity (based on a Clausius-Mosotti summation of ionic polarizabilites) is attributed by a number of authors to a "rattling" effect of the displaced cation and local variations in the Lorentz field ${ }^{36-38}$

The Fe-O-Fe angles were also reviewed, which shows that the angles are becoming equivalent at temperatures corresponding to the maximum in the relative permittivity (Figure 10). These changes in Fe-O-Fe angles are influenced by changes in the degree of octahedral tilting as well as the distortion in the octahedra, which are both important effects influencing the overall polarisation. However, it is more instructive to look at structural effects in terms of two distinct types of symmetry modes, with the tilt modes being discussed below.

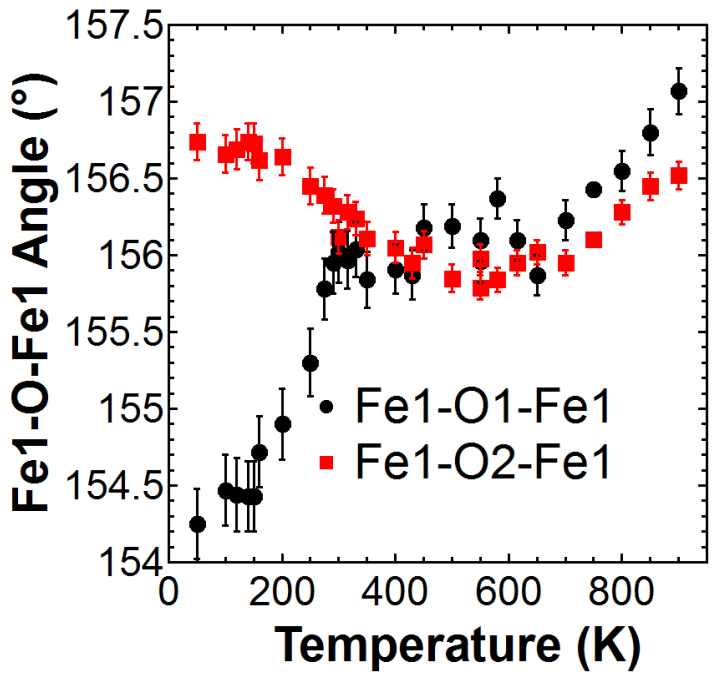

Figure 10. Changes in the Fe-O-Fe angles with temperature, which are linked to the changes in the two octahedra tilt modes and the octahedral distortion.

\section{Structural response to magnetic ordering}

As shown in Figure 3 the reduced lattice parameters show an increase in the orthorhombic distortion, $D_{o}$, with increasing temperature with a broad maximum in the range $c a$. 650-700 $\mathrm{K}$ (Figure 4). This change in distortive behaviour correlates with the paramagnetic - antiferromagnetic transition and the onset of a magnetostrictive effect.

The paramagnetic to antiferromagnetic transition in $\mathrm{Bi}_{1}$ ${ }_{x} \mathrm{La}_{x} \mathrm{FeO}_{3}$ has been studied for compositions $0.18 \leq \mathrm{x} \leq 0.35$. $^{3}$ The authors detected a weak anomaly near 670-675 K using DSC and suggested it was associated with an AFM to paramagnetic phase transition that has been reported elsewhere by Roginskaya and Polomska. ${ }^{3,16,17}$ The onset of AFM order in BLFO50 occurs slightly higher circa $700 \mathrm{~K}$, as indicated by the fit of the magnetic data (Figure 2) and the presence of a predominantly magnetic reflection in the neutron diffraction data at $4.55 \AA$ d-spacing (Supporting Information, Figure S3). This onset of magnetic order influences the orthorhombic distortion, as described in more detail below. 


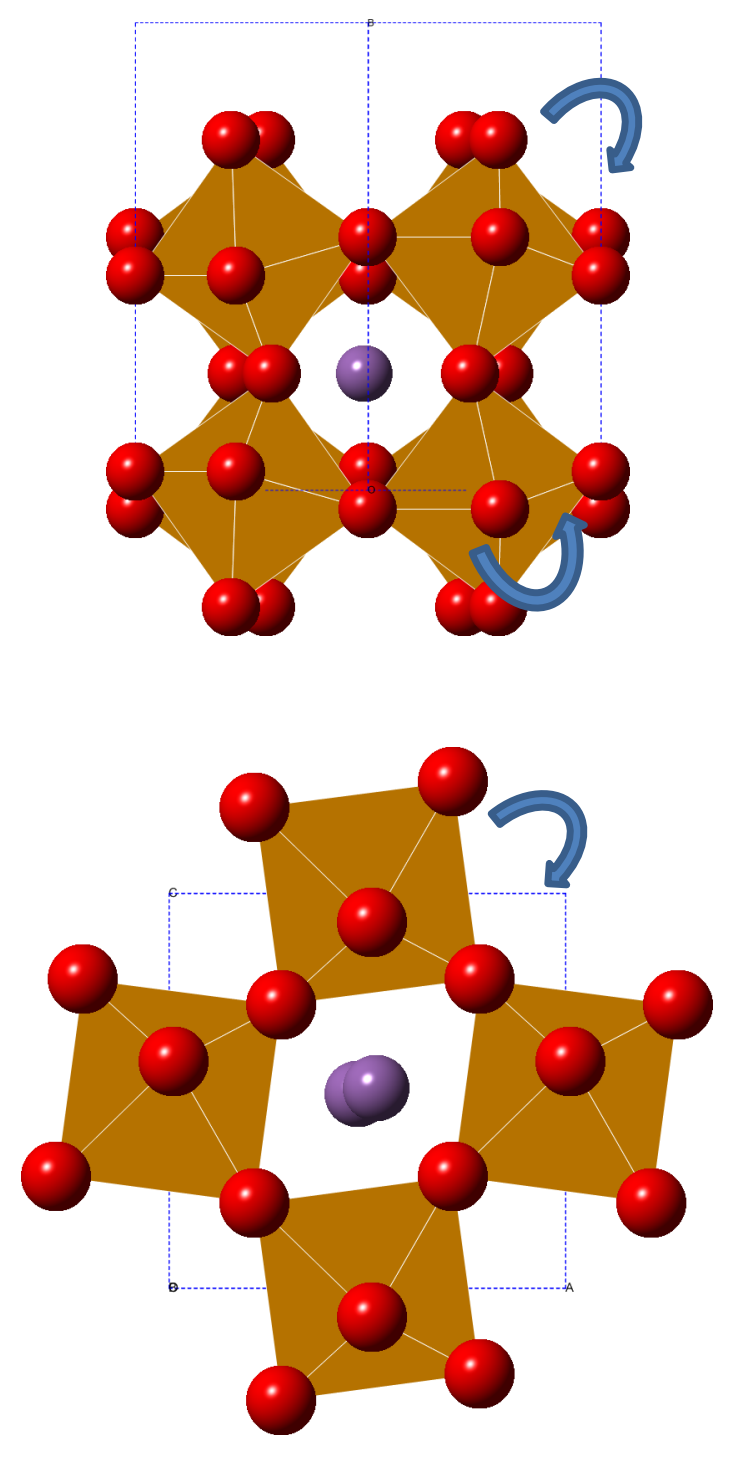

Figure 11. Anti-phase (R) rotation about [101] (top) and the In-phase (M) rotation about [010], (bottom) with respect to the Pnma cell.

The initial approach studied changes in the Fe-O-Fe angles. These angles are influenced by the two possible tilting modes, the in-phase $\left(\mathrm{M}_{3}^{+}\right)$and anti-phase $\left(\mathrm{R}_{4}^{+}\right)$tilts shown in Figure 11. The Fe-O1-Fe angle is influenced by the anti-phase tilt whereas the $\mathrm{Fe}-\mathrm{O} 2-\mathrm{Fe}$ angle is influenced by both tilts. An increasing Fe-O-Fe angle suggests a decreasing octahedral tilt, neglecting minor octahedral distortion effects. The increase in the Fe-O1-Fe angle up to $\mathrm{ca}$. $300 \mathrm{~K}$ therefore suggests a decrease in the anti-phase tilt, while the decrease in the Fe-O2$\mathrm{Fe}$ angle suggests an increase in the in-phase tilt. The Fe-O-Fe angles (Figure 10) also suggest that there is a significant change at $\mathrm{ca} .700 \mathrm{~K}$ where both the Fe-O-Fe angles start to increase with temperature. However, the Fe-O-Fe angles are also influenced by distortions of the octahedra i.e., both tilts decrease above this temperature. It is therefore necessary to study the separate influences of each of the tilts on the Fe-OFe angles.

With increasing temperature the anti-phase tilt $\left(\mathrm{R}_{4}{ }^{+}[\mathrm{O}]\right.$ mode) decreases, as expected, while the in-phase tilt $\left(\mathrm{M}_{3}{ }^{+}[\mathrm{O}]\right.$ mode) increases with temperature reaching a broad maximum round $650-750 \mathrm{~K}$ (Figure 12 ). The increase in the inphase tilt as a function of temperature is highly unusual and has only been observed as a very weak effect over a limited temperature range, for example in $\mathrm{KCaF}_{3}{ }^{39}$ The effect in this case is considerably larger and occurs over a more extensive temperature range.

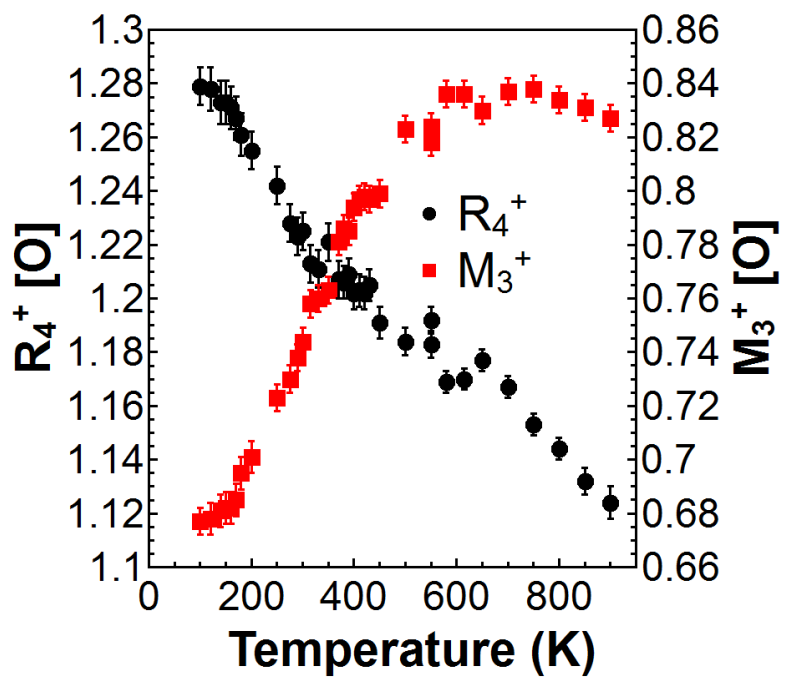

Figure 12. ISODISTORT tilt modes as a function of temperature showing the in-phase rotation about [010] $\left(\mathrm{M}_{3}^{+}\right)$and anti-phase tilt $\left(\mathrm{R}_{4}{ }^{+}\right)$about [101].

The high-temperature plateau in $\mathrm{M}_{3}{ }^{+}$appears at first-sight approximately in-line with the maximum in the orthorhombic distortion (Figure 4). However, the correlation is not exact and, although the in-phase tilt is obviously linked to the orthorhombic distortion it cannot be, in itself, the cause of the distortion (a strictly rigid in-phase tilt would leave the lattice metrically tetragonal, but destroys the crystallographic 4-fold axis). Instead, a non-zero $D_{o}$ parameter is typically associated with A-site cation displacements in $\mathrm{GdFeO}_{3}$-type perovskites. ${ }^{40}$ In the $\mathrm{Bi}_{1-x} \mathrm{La}_{x} \mathrm{FeO}_{3}$ system, a number of studies have examined the $A$-site behaviour and have shown that the structural features are closely associated with A-site displacements. ${ }^{3,12}$ The displacement of the A-site cation along the a-axis is represented in ISODISTORT by the $\mathrm{X}_{5}{ }^{+}$[A site] mode (Figure 13). The $X_{5}{ }^{+}$mode increases up to ca. $650 \mathrm{~K}$ where it reaches a maximum before falling with temperature. This correlates strongly with the orthorhombic distortion (Figure 13). This clearly shows that displacements on the Asite cause the increasing orthorhombic distortion, with a secondary correlation being the increase in the $\mathrm{M}_{3}{ }^{+}$in-phase tilt. 
The $\mathrm{X}_{5}{ }^{+}$symmetry mode also has a much smaller oxygen displacement contribution (Supporting Information, Figure S6).

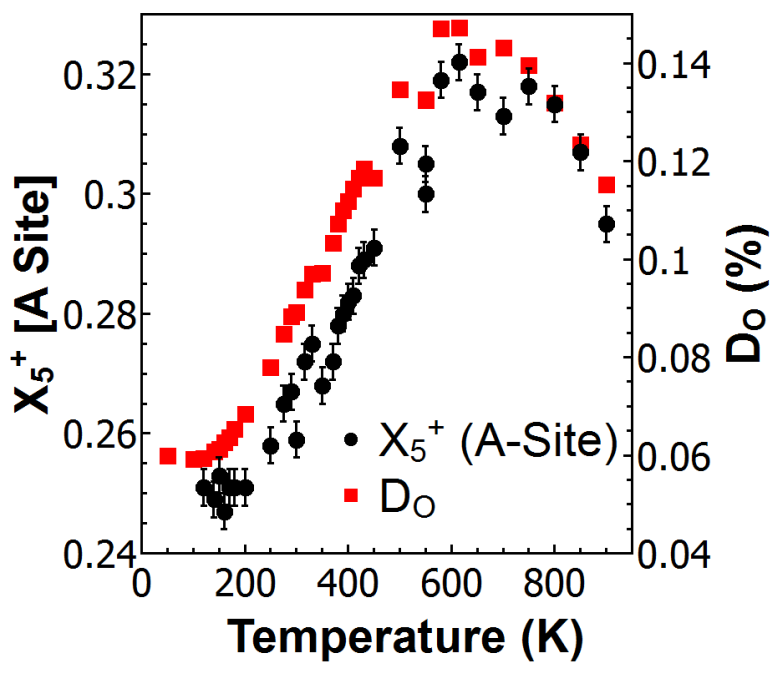

Figure 13. ISODISTORT $\mathrm{X}_{5}{ }^{+}$[A site] displacement mode as a function of temperature describing A-cation displacements along the a-axis and the correlation with orthorhombic strain, $D_{o}$.

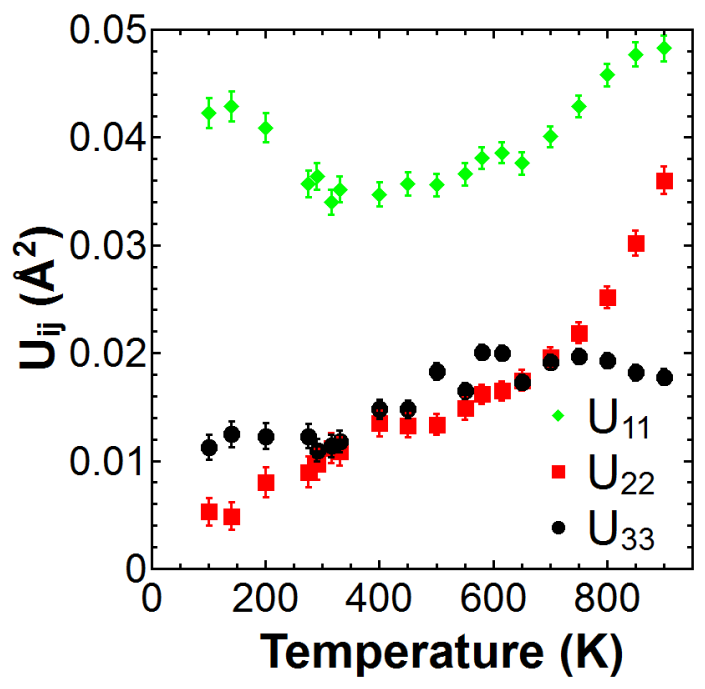

Figure 14. Anisotropic atomic thermal displacement parameters $\left(U_{i j}\right)$ as a function of temperature along a $\left(U_{11}\right), b\left(U_{22}\right)$, and $c$ $\left(U_{33}\right)$ axes.

The structural models so far have considered the bismuth and lanthanum sites as equivalent. Although $\mathrm{Bi}^{3+}$ and $\mathrm{La}^{3+}$ have similar ionic radii when comparing equivalent coordination numbers ${ }^{41}$ the effect of the $\mathrm{Bi}^{3+}$ lone pair has not been considered (The 8-coordinate $\mathrm{Bi}^{3+}$ and $\mathrm{La}^{3+}$ ionic radii according to Shannon are $1.170 \AA$ and $1.160 \AA$, respectively). ${ }^{41}$ The relative displacements of $\mathrm{Bi}^{3+}$ and $\mathrm{La}^{3+}$ away from the refined, 'averaged' A-site position are likely to be slightly different. These local displacements in the A-site will be evident in the atomic displacement parameters. If the atom positions are refined anisotropically the direction of the most significant relative displacement can be inferred. Anisotropic refine- ments of the A-site confirm that there is indeed a relative displacement of the two cations, and show that the $U_{11}$ parameter is significantly larger than $U_{22}$ and $U_{33}$ (Figure 14). The $U_{11}$ parameter corresponds to the displacement along the a-axis of the Pnma unit cell. This suggests that the bismuth and lanthanum sites are not equivalent and are displaced along the a-axis but by differing degrees. This displacement direction is consistent with the change in the orthorhombic distortion $\mathrm{X}_{5}{ }^{+}$mode which shows the overall displacement in the a-axis based on isotropic refinements (Figure 13). The $\mathrm{X}_{5}{ }^{+}$ mode tracks with the orthorhombic distortion, representing the average displacement of the lanthanum and bismuth site, which it is now possible to demonstrate, is due in part to relative displacements of the two cations away from a single Asite.

The structural analysis so far has shown a displacement of the A-site cations along the a-axis, where the local positions of $\mathrm{La}$ and $\mathrm{Bi}$ are not perfectly equivalent. This A-site displacement drives the anomalous change in the orthorhombic distortion, with the secondary effect of the abnormal in-phase tilt behaviour. However, the underlying cause of the invariant c lattice parameter below $\sim 650 \mathrm{~K}$ has not yet been addressed. The caxis corresponds with the direction of the net magnetisation. This is linked to a magnetostrictive effect within the material. On cooling from the paramagnetic region the sample orders antiferromagnetically at $\mathrm{T}_{\mathrm{N}} \sim 704 \mathrm{~K}$. Eventually the net magnetisation increases to such a point where it has a detectable effect on the lattice parameters such that there is a deviation in the c-axis from the expected near-linear thermal contraction (as observed in the a- and b-axes) to temperature invariant behaviour, the so-called "Invar effect". ${ }^{42}$ A similar effect has been observed in other perovskites such as $\mathrm{LaTiO}_{3}$ which adopts the $\mathrm{GdFeO}_{3}$ structure (Pbnm) where with the onset of antiferromagnetic $\left(G_{x}\right)$ order results in an increase in the orthorhombic splitting with a changing $\sqrt{ } 2$ axis set. ${ }^{43}$ Magnetostrictive effects have also been observed with various ruthenate based perovskites. ${ }^{42,44,45}$

Typically, a Deybe-Grüneisen approximation can be applied to the unit cell volume to determine the contraction of the unit cell with decreasing temperature. This accounts for the internal energy of the system and models the volume change with temperature giving the hypothetical contraction of the unit cell in the paramagnetic state. The relative difference between the unit cell parameters or volume of the hypothetical paramagnetic state and that observed experimentally gives the contribution of the magnetostrictive effect. To do this fit an estimation of the Grüneisen parameter needs to be determined from measurements of the heat capacity, bulk modulus and volume expansion. ${ }^{46}$ As a simple alternative, we have approximated the thermal expansivity of the unit cell parameters with a second order polynomial, $y=A_{2} T^{2}+$ $A_{1} T+A_{0}$. The $P n^{\prime} m a^{\prime}$ magnetic space group contains no magnetic component along $\mathrm{x}$, and so the a lattice parameter was fitted over the entire temperature range ( $50 \mathrm{~K}$ to $900 \mathrm{~K}$ ). The polynomial with $\mathrm{A}_{2}=2.2174 \times 10^{-8}, \mathrm{~A}_{1}=2.0900 \times 10^{-5}$ and $A_{0}=3.9338$ gave a good fit to the observed data, figure 3 . The b- and c-axes were also fitted to this polynomial with the $A_{2}$ 
and $A_{1}$ coefficients fixed and $A_{0}$ allowed to vary; the b-axis was fitted over the entire temperature range but the c-axis was only fitted in the near-linear, high temperature region and then extrapolated to lower temperatures, Figure 3. $A_{0}=$ 3.9240 and 3.9127 for b- and c-axes, respectively.

The difference between the predicted contraction and the experimental values, Figure 3, represents the contribution from magnetostriction. The magnitude of magnetostrictive strain $\left(\omega_{\mathrm{x}}\right)$ of the lattice can be calculated as:

$$
\omega_{x}=\frac{x_{\text {exp }}-x_{\text {calc }}}{x_{\text {calc }}}
$$

where $x_{\exp }$ and $x_{\text {calc }}$ are the experimentally determined and calculated lattice parameters, respectively. The temperature dependence of magnetostriction is shown in Figure 15, and clearly indicates an increasing c-axis strain with decreasing temperature, reaching ca. $0.3 \%$ at $50 \mathrm{~K}$, which is of a similar magnitude to other magnetostrictive oxides. ${ }^{42,44,45}$. There is no detectable strain in the other axes.

In summary, BLFO50 adopts the orthorhombic $\mathrm{GdFeO}_{3}$ crystal structure described by Pnma symmetry. On cooling from high temperature it magnetically orders to a G-type antiferromagnet $\left(T_{N} c a .704 \mathrm{~K}\right.$ ) with the moment along the c-axis (Shubnikov symmetry $\left.P n^{\prime} m a^{\prime}\right)$. The magnetic ordering results in: (a) a magnetostrictive (Invar) effect along the magnetic c axis; (b) antipolar A-site displacements along the a axis; and (c) contrasting changes in in-phase and anti-phase octahedral tilts. The net result is a strong orthorhombic distortion which decreases on further cooling. At $c a .300 \mathrm{~K}$ the A-site displacements and a distortion of the octahedra caused by a scissoring of the axial oxygens results in a change in the dielectric permittivity from ca. 250 to 180 .

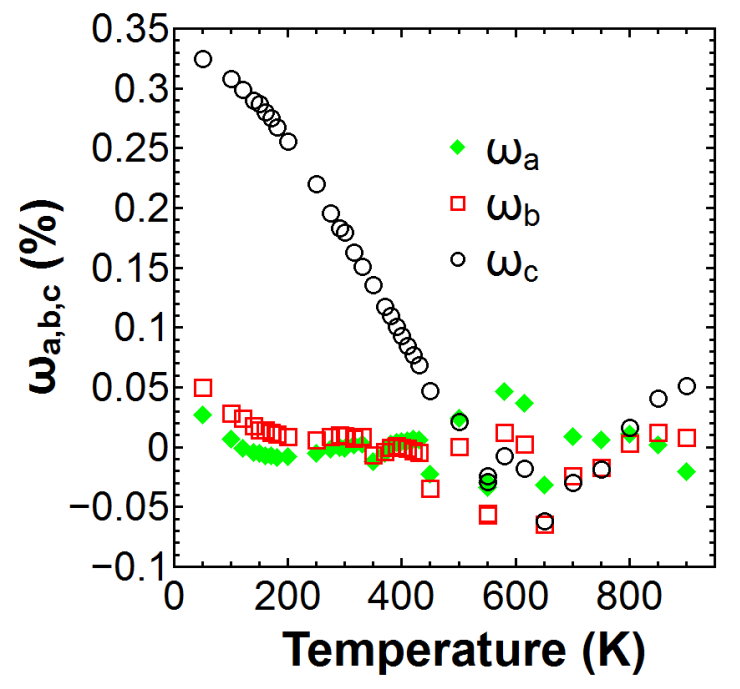

Figure 15. Magnetostrictive contribution $\left(\omega_{x}\right)$ as a function of temperature showing increasing strain in the c-axis below the Neel temperature.

\section{CONCLUSIONS}

A combination of electrical and structural analysis using temperature dependent powder neutron diffraction has shown that lanthanum doped $\mathrm{BiFeO}_{3}$ of composition $\mathrm{Bi}_{0.5} \mathrm{La}_{0.5} \mathrm{FeO}_{3}$ is a wide band gap semiconductor with its structural and dielectric behaviour driven by the magnetic properties. The detailed crystallographic changes (structural distortions) were analysed using both conventional Rietveld analysis and also the symmetry mode ISODISTORT tool. The analysis shows that the material adopts the $\mathrm{GdFeO}_{3}$ structure and that there is an unusual orthorhombic distortion with an increasing in-phase tilt as a function of increasing temperature. This distortion has a corresponding antipolar atomic displacement of the Asite where the lanthanum and bismuth are displaced by different degrees along the a-axis. The orthorhombic distortion is caused by the onset of antiferromagnetic order $\left(T_{N} \sim 750 \mathrm{~K}\right)$ and a magnetostrictive Invar effect along the magnetic c-axis. The magnetically driven tilt combines with distortion of the octahedra and a corresponding A-site displacement along the $c$-axis resulting in a change in the dielectric permittivity at $c a$. $300 \mathrm{~K}$. The use of symmetry-mode analysis (via ISODISTORT), in addition to conventional crystallographic analysis, presents a new paradigm for investigation of structure-property relationships in lanthanide doped $\mathrm{BiFeO}_{3}$.

\section{ASSOCIATED CONTENT}

Supporting Information contains: details of Rietveld refinements of neutron data including magnetic contributions; internal octahedral bond angles; additional impedance spectra, ISODISTORT oxygen displacement mode as a function of temperature. This material is available free of charge via the Internet at http://pubs.acs.org.

\section{AUTHOR INFORMATION}

\section{Corresponding Author}

Email: finlay.morrison@st-andrews.ac.uk

\section{ACKNOWLEDGMENT}

The authors would like to thank the Royal Society for a University Research Fellowship (FDM), Engineering and Physical Sciences Research Council for a studentship (CMK), and the Science and Technology Facilities Council for access to neutron facilities.

\section{REFERENCES}

(1) Catalan, G.; Scott, J. F. Adv. Mater. 2009, 21, 2463.

(2) Eerenstein, W.; Morrison, F. D.; Dho, J.; Blamire, M. G.; Scott, J. F.; Mathur, N. D. Science 2005, 307, 1203.

(3) Rusakov, D. A.; Abakumov, A. M.; Yamaura, K.; Belik, A. A.; Van Tendeloo, G.; Takayama-Muromachi, E. Chem. Mater. 2011, 23, 285.

(4) Troyanchuk, I. O.; Bushinsky, M. V.; Karpinsky, D. V.; Mantytskaya, O. S.; Fedotova, V. V.; Prochnenko, O. I. Phys. Status Solidi B 2009, 246, 1901.

(5) González-Vázquez, O. E.; Wojdeł, J. C.; Diéguez, O.; Íñiguez, J. Phys. Rev. B 2012, 85, 064119.

(6) Troyanchuk, I. O.; Karpinsky, D. V.; Bushinsky, M. V.; Khomchenko, V. A.; Kakazei, G. N.; Araujo, J. P.; Tovar, M.; Sikolenko, V.; Efimov, V.; Kholkin, A. L. Phys. Rev. B 2011, 83, 054109.

(7) Troyanchuk, I. O.; Bushinsky, M. V.; Karpinskii, D.; Mantytskaya, O.; Fedotova, V.; Prokhnenko, O. JETP Letters 2008, 87, 641.

(8) Karimi, S.; Reaney, I. M.; Levin, I.; Sterianou, I. Appl. Phys. Lett. 2009, 94, 112903. 
(9) Levin, I.; Karimi, S.; Provenzano, V.; Dennis, C. L.; Wu, H.; Comyn, T. P.; Stevenson, T. J.; Smith, R. I.; Reaney, I. M. Phys. Rev. B 2010, 81, 020103.

(10) Levin, I.; Tucker, M. G.; Wu, H.; Provenzano, V.; Dennis, C. L.; Karimi, S.; Comyn, T.; Stevenson, T.; Smith, R. I.; Reaney, I. M. Chem. Mater. 2011, 23, 2166.

(11) Glazer, A. M.; Roleder, K.; Dec, J. Acta Crystallogr. Sect. B: Struct. Sci. 1993, 49, 846.

(12) Lee, J. H.; Choi, H. J.; Lee, D.; Kim, M. G.; Bark, C. W.; Ryu, S.; Oak, M. A.; Jang, H. M. Phys. Rev. B 2010, 82, 045113.

(13) Sosnowska, I.; Neumaier, T. P.; Steichele, E. J. Phys. C 1982, 15, 4835. (14)Moreau, J. M.; Michel, C.; Gerson, R.; James, W. J. J. Phys. Chem. Solids 1971, 32, 1315.

(15) Le Bras, G.; Bonville, P.; Colson, D.; Forget, A.; Genand-Riondet, N.; Tourbot, R. Physica B 2011, 406, 1492.

(16) Yu. E Roginskaya; Yu. E Venevtsev; S. A Fedotov; G. S. Zhdanov Sov. Phys. Crystallogr. 1964, 8, 490.

(17) Polomska, M.; Kaczmarek, W.; Pajak, Z. Phys. Status Solidi 1974, 23, 567.

(18) Peterlin-Neumaier, T.; Steichele, E. J. Magn. Magn. Mater. 1986, 59, 351.

(19) Acharya, S.; Mondal, J.; Ghosh, S.; Roy, S. K.; Chakrabarti, P. K. Mater. Lett. 2010, 64, 415.

(20) Treves, D. J. Appl. Phys. 1965, 36, 1033.

(21) Marezio, M.; Remeika, J. P.; Dernier, P. D. Acta Crystallogr. Sect. B: Struct. Sci. 1970, 26, 2008.

(22) Arnold, D. C.; Knight, K. S.; Catalan, G.; Redfern, S. A. T.; Scott, J. F.; Lightfoot, P.; Morrison, F. D. Adv. Funct. Mater. 2010, 20, 2116.

(23) Arnold, D. C.; Knight, K. S.; Morrison, F. D.; Lightfoot, P. Phys. Rev. Lett. 2009, 102, 027602.

(24) Geller, S.; Wood, E. A. Acta Cryst. 1956, 9, 563.

(25) Simões, A. Z.; Garcia, F. G.; Riccardi, C. d. S. Mater. Chem. Phys. 2009, 116, 305.

(26) Pandit, P.; Satapathy, S.; Gupta, P. K. Physica B 2011, 406, 2669.

(27) Larson, A. C.; Von Dreele, R. B. General Structure Analysis System (GSAS) Los Alamos National Laboratory Report LAUR 86-748, 1994.

(28) Toby, B. J. Appl. Crystallogr. 2001, 34, 210.

(29) White, R. L. J. Appl. Phys. 1969, 40, 1061.

(30) Kidner, N. J.; Perry, N. H.; Mason, T. O.; Garboczi, E. J. J. Am. Ceram. Soc. 2008, 91, 1733.

(31) Irvine, J. T. S.; Sinclair, D. C.; West, A. R. Adv. Mater. 1990, 2, 132.

(32) West, A. R.; Sinclair, D. C.; Hirose, N. J. Electroceram. 1997, 1, 65.

(33) Kalantari, K.; Sterianou, I.; Karimi, S.; Ferrarelli, M. C.; Miao, S.; Sinclair, D. C.; Reaney, I. M. Adv. Funct. Mater. 2011, 21, 3737.

(34) Islam, M. S. J. Mater. Chem. 2000, 10, 1027.

(35) Campbell, B. J.; Stokes, H. T.; Tanner, D. E.; Hatch, D. M. J. Appl. Crystallogr. 2006, 39, 607.

(36) Shannon, R. D. J. Appl. Phys. 1993, 73, 348.

(37) Parker, R. A. Phys. Rev. 1961, 124, 1713.

(38) Roberts, S. Phys. Rev. 1951, 81, 865.

(39) Knight, K. S.; Darlington, C. N. W.; Wood, I. G. Powder Diffr. 2005, 20, 7.

(40) Woodward, P. Acta Crystallogr. Sect. B: Struct. Sci. 1997, 53, 44.

(41) Shannon, R. D. Acta Crystallogr. Sect. A: Found. Crystallogr. 1976, 32, 751.

(42) Kiyama, T.; Yoshimura, K.; Kosuge, K.; Ikeda, Y.; Bando, Y. Phys. Rev. B 1996, 54, R756.

(43) Komarek, A. C.; Roth, H.; Cwik, M.; Stein, W. D.; Baier, J.; Kriener, M.; Bourée, F.; Lorenz, T.; Braden, M. Phys. Rev. B 2007, 75, 224402.

(44) Dabrowski, B.; Avdeev, M.; Chmaissem, O.; Kolesnik, S.; Klamut, P. W.; Maxwell, M.; Jorgensen, J. D. Phys. Rev. B 2005, 71, 104411.

(45) Ranjan, R.; Senyshyn, A.; Garg, R.; Boysen, H. J. Appl. Phys. 2011, 109, 073908.

(46) Knight, K. J. Electroceram. 2011, 27, 143. 


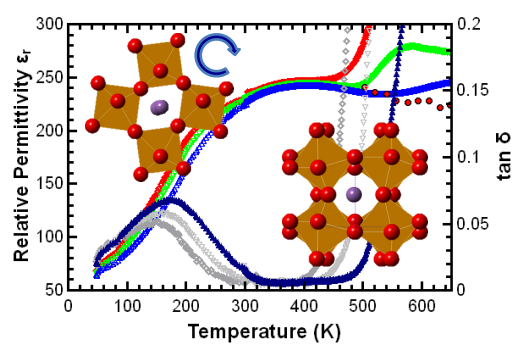

